\title{
EVALUATION OF LONG-TERM BEIDOU/GPS OBSERVATION QUALITY BASED ON G-NUT/ANUBIS AND INITIAL RESULTS
}

\author{
Lewen ZHAO ${ }^{1,2)}$, Jan DOUSA ${ }^{2)}$, Pavel VACLAVOVIC ${ }^{2)}$, Shirong YE ${ }^{1) *}$ and Fengyu XIA ${ }^{1)}$ \\ ${ }^{1)}$ GNSS Research Center, Wuhan University, 129 Luoyu Road, Wuhan 430079, China \\ 2) New Technologies for the Information Society, Research Institute of Geodesy, Topography and Cartography, Zdiby, Czech Republic
}

*Corresponding author's e-mail: srye@whu.edu.cn

\begin{tabular}{l} 
ARTICLE INFO \\
\hline Article history: \\
Received 16 January 2018 \\
Accepted 16 March 2018 \\
Available online 22 March 2018 \\
\hline
\end{tabular}

\section{Keywords:}

G-Nut/Anubis

Pre-processing

BeiDou

Observation quality

Open source

\begin{abstract}
Reliable observations are the prerequisite for high-precision GNSS data processing. Data quality evaluation at the pre-processing procedure can help the user to identify the weakness of the observations. The open source software package G-Nut/Anubis developed at Geodetic Observatory Pecny (GOP) is introduced and used for the evaluation of long-term BeiDou/GPS signal. Several key indicators are selected to evaluate the BeiDou/GPS observations. Quantitative analysis shows that more than 100 stations can achieve 6-hour standalone BeiDou positioning at least. The completeness of the BeiDou phase and code observations at three frequencies is higher than that of the GPS observations in the selected station which is mainly attributed to the high elevation observations from GEO and IGSO satellites. Qualitative analysis shows that the multipath effects for pseudorange observations are correlated with signal, receiver types and firmware versions besides the station environment. Users attempting to improve the pseudorange observations by multipath filter should pay attention also to the receiver-specific settings. Cycle slip counts are used to indicate the quality of carrier-phase observations. Results show that BeiDou C05 satellite is more affected by the cycle slips, in particular the third frequency observables. The GPS third frequency obsevables are more robust compared to the legacy frequencies. Comprehensive single point positioning results show that positioning accuracy for BeiDou is worse than that of GPS, which is mainly caused by less active satellites, worse geometry and larger errors in the broadcast ephemeris. The BeiDou positioning accuracy was further degraded by the $\mathrm{C} 13 / \mathrm{C} 15$ satellite and dominated by large clock errors. Broadcast evaluation shows that the BeiDou orbit errors are related to the constellations. Overall, the orbit precision for both GPS and BeiDou has been gradually improved. Outliers were observed for navigation records assigned with the healthy status, especially for BeiDou broadcast orbits.
\end{abstract}

\section{INTRODUCTION}

The quality of the Global Navigation Satellite System (GNSS) observation data is a key factor that affects the high-precision applications. Poor GNSS observations can usually be identified with two separate procedures: (1) data quality monitoring and editing based on the raw observations at preprocessing procedure; (2) outlier detection based on pre-fit and post-fit residuals in the parameters estimation procedure. The outlier detection usually requires precise modeling of signal propagation errors and several iterations to reject gross errors, thus can be time-consuming for the network processing. Besides, the observations might be affected by environmental multipath, receiver-specific hardware anomalies or satellite-specific outages. Post-fit residual checking procedure cannot identify the causes of such poor observations sufficiently, and gross errors can significantly impact the solution convergence. Therefore, the evaluation of GNSS data quality at the pre-processing level is important to identify receiver-, environmental- and satellitespecific problems affecting the observations.

Several quality control programs, i.e. TEQC (Translation, Editing and Quality Checking) (Estey and Meertens, 1999), GFZRNX (Nischan, 2016) and G-Nut/Anubis (Vaclavovic and Dousa, 2015a) are now available for multi-GNSS observations. The TEQC was originally supported by UNAVCO for GPS data quality checking. It allows the data translation from the binary format to the RINEX (Receiver INdependent EXchange) format, metadata extraction, editing, and correction of RINEX files, as well as a simple quality check function. With the availability of new GPS signals and additional GNSS constellations (Galileo, GLONASS, BeiDou, QZSS, SBAS, IRNSS), the International GNSS service (IGS) has introduced the version 3 of the RINEX format. Unfortunately, TEQC cannot support RINEX 3.XX format observations. GFZRNX toolbox, developed by GFZ, supports all new GNSS data and constellations in the RINEX 3.XX format, however, with the main 
focus on data editing and translation. Though preprocessing can be achieved with the cooperation of GFZRNX and TEQC, however, no comprehensive statistical indicators about the quality of all observations, frequencies, tracking modes, and systems are provided. Moreover, the two packages are not open sources; thus users cannot modify the code according to their demands.

The G-Nut core library has been developed by the Geodetic Observatory Pecny (GOP) with a focus on a deep expertize in multi-GNSS data processing for updating and enhancing various GOP scientific products. Different advanced applications, including real-time troposphere monitoring (Douša et al., 2018), numerical weather modeling (Dousa and Elias, 2014) and precise clock estimation (Ye et al., 2018) have been developed on the basis of the library. The GNut/Anubis software is an open-source package derived from the G-Nut library in support of GNSS data quality control. The software supports all multiGNSS observations and navigation data from RINEX 3.XX and 2.XX formats as well as precise ephemeris from the SP3 format. The data quality checking is performed at three different levels, i.e. quantitative, qualitative and complex control (Václavovic and Dousa, 2013; Václavovic and Dousa, 2015).

The quantitative control characterizes the format correctness, consistency of actual data content with respect to records in the file header. Observation header records provide metadata for users to identify the file content at a first glance, whereas an actual completeness of data content is important for real user solutions. The benefits of multi-frequency observations on fast Precise Point Positioning (PPP, Zumberge and Heflin et al., 1997) ambiguity resolution and PPP re-convergence have been demonstrated (Geng and Bock, 2013; Li and Wang et al., 2014). Lack of the observations of a specific frequency will degrade the user solutions. Therefore, quantitative indicators help users to identify the actual availability of file observations.

The qualitative control requires specific algorithms, which can give users advanced insight into the quality of code and phase observations. In GNSS, pseudorange multipath can seriously degrade the instant accuracy of applications that rely on pseudorange observables, including differential pseudorange navigation, and ionospheric monitoring. Compared to the pseudorange observations, the carrier phase observations are generally used in the high precision applications, but the phase signals can be significantly affected by presence of cycle slips. Multipath effects are mainly caused by the satellite signals reflected from nearby objects such as buildings, cars, walls, and from the site environment including vegetation, the earth ground, water or other surfaces. Cycle slips are usually caused due to the loss of lock on a satellite signal, power loss, low SNR, and high level of ionospheric activity. The statistical results on the two indicators can help to identify the signal- and environmental-specific factors that might affect GNSS positioning.

The all aforementioned indicators are based on the single satellite observation statistics. For precise applications, a minimum of four satellites is required for a single GNSS positioning. To demonstrate the comprehensive quality of observations, results from standard point positioning (SPP) is used as a complex indicator. Note that the SPP results also strongly depend on the broadcast ephemeris. Therefore, indicators from SPP evaluation can additionally reflect a quality of navigation message, a primary element of GNSS system providing essential information for user PNT (Positioning, Navigating and Timing) applications. Currently, the evaluation of the quality of GNSS observations (Kim and Seo et al., 2014) and broadcast data (Nicolini and Caporali, 2018) are based on a short period only, where longterm trends and variations cannot be detected. The main objective of this study is utilizing selected indicators provided by the G-Nut/Anubis to analyze a long-term quality of BeiDou and GPS observations. The causes that lead to the indicator changes are discussed in detail. By analyzing the series of the indicators, we compare the performance of BeiDou and GPS observation signals and derive conclusions.

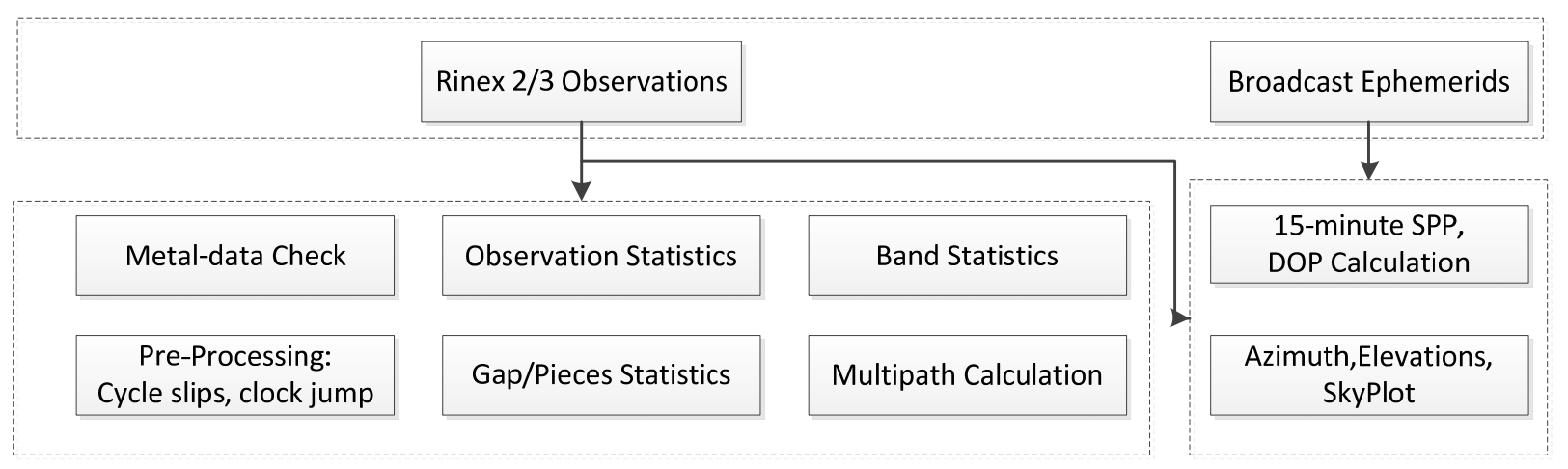

Fig. 1 Framework of the process for G-Nut/Anubis software. 


\section{MODEL AND METHOD}

Figure 1 shows the framework of the whole process using the G-Nut/Anubis software. Anubis can be started using a configuration file in XML format. The inputs mainly contain the RINEX 2.XX/3.XX format observation files and the broadcast ephemeris. The main features include the statistical indicators from the raw GNSS observations and the calculation of satellite elevations, azimuths, and SPP if constellation-specific navigation messages or SP3 products are available. The entire statistics are reported in the summary file with different verbosity, which can be used for further qualitative analysis.

\section{DATA AVAILABILITY INDICATOR}

Knowledge about the availability of observation data is the pre-condition for any GNSS applications. The G-Nut/Anubis software provides statistical indicators about valid observations in the selected stations. The availability indicator is defined as $R_{\text {avai }}$ :

$$
R_{\text {avai }}=\frac{U s e E p}{\operatorname{ExpEp}}
$$

where 'ExpEp' indicates the number of expected epochs when calculated using pre-defined data interval and data sampling and, 'UseEp' indicates the number of usable epochs supported with at least 4 satellites in the epoch with dual-frequency data for each GNSS constellation. From a huge number of GNSS tracking stations, tracking different GNSS signals when equipped with receivers of different manufacturers, the indicator can facilitates users to select efficiently stations of interest.

\section{DATA COMPLETENESS INDICATOR}

Apart from the availability of observations, the completeness of observations at individual bands is an important indicator for a continuous estimation of parameters in GNSS applications. The completeness of data is defined by the ratio $R_{\text {comp }}$ given in Eq. (2):

$$
R_{\text {comp }}=\frac{H a v O b s}{\operatorname{ExpObs}}
$$

where 'ExpObs' indicates the number of expected observations at a specific band and signal, calculated using the observation period and data sampling and, 'HavObs' indicates the actual number of records in the observation file. Theoretically, satellite signals should be continuously tracked from all epochs when the satellite is in view, which would be theoretically indicated by $100 \%$ completeness. However, due to environmental and instrumental effects, mainly signals from satellites at low elevations are suffering from various interruptions. The completeness ratio at different elevation mask can then illustrate issues with low-elevation tracking. The elevation mask is usually set to above 5 degrees for precise positioning
(Langley, 1999). Low observations still significantly contribute to the decorrelation of simultaneously estimated tropospheric parameters and station height and improve the modelling of tropospheric asymmetry (Kačmařík et al., 2017), both improving the repeatability of station coordinates (Douša et al., 2017).

\section{MULTIPATH INDICATOR}

The multipath effect refers to errors due to the indirect-path of satellite signals recorded by a GPS receiver, typically from the signal reflected by the surroundings of the receiver antenna, and thus differing from the direct-path signal. Multipath effects are often considered as stochastic errors; they differ according to the characteristics of the reflecting surfaces and are difficult to correct. The multipath linear combination is constructed using a singlefrequency code measurement and dual-frequency phase measurements, which can be expressed as (Estey and Meertens, 1999)

$$
\begin{aligned}
& M P_{1}=P_{1}-\frac{f_{1}^{2}+f_{2}^{2}}{f_{1}^{2}-f_{2}^{2}} L_{1}+\frac{2 f_{2}^{2}}{f_{1}^{2}-f_{2}^{2}} L_{2} \\
& M P_{2}=P_{2}-\frac{2 f_{1}^{2}}{f_{1}^{2}-f_{2}^{2}} L_{1}+\frac{f_{1}^{2}+f_{2}^{2}}{f_{1}^{2}-f_{2}^{2}} L_{2} \\
& M P_{3}=P_{3}-\frac{2 f_{1}^{2}}{f_{1}^{2}-f_{3}^{2}} L_{1}+\frac{f_{1}^{2}+f_{3}^{2}}{f_{1}^{2}-f_{3}^{2}} L_{3}
\end{aligned}
$$

where $P_{i}$ and $L_{i}(\mathrm{i}=1,2,3)$ are the code and carrier phase measurements, respectively; $f_{i}$ denotes the frequency for carrier phase. For the G-Nut/Anubis software, a generic triple-frequency combination for multipath estimates has been developed (Václavovic and Douša, 2016) for more flexible multi-frequency data processing. The systematic errors and noise of carrier phase measurements are assumed to be negligible compared with that of code observations. It should be mentioned that the multipath combination contains a constant ambiguity term of combined carrier phases. The ambiguity term can be smoothed and deduced from the multipath series if there are no cycle slips. To numerically indicate the magnitude of the multipath on a specific band and specific GNSS, the root mean square of the multipath series are calculated and providing an indicator in the summary files of G-Nut/Anubis.

\section{CYCLE SLIPS AND SINGLE POINT POSITIONING INDICATORS}

Cycle slip counts can be used as an indicator for the quality of phase observations, but the counts are highly dependent on the implemented methods. The triple-frequency cycle slip detection method proposed by Zhao et al. (2015) has been implemented in the package. Besides, Vaclavovic and Douša (2016) presented a detailed description on the method implemented in the package; interested users can refer 


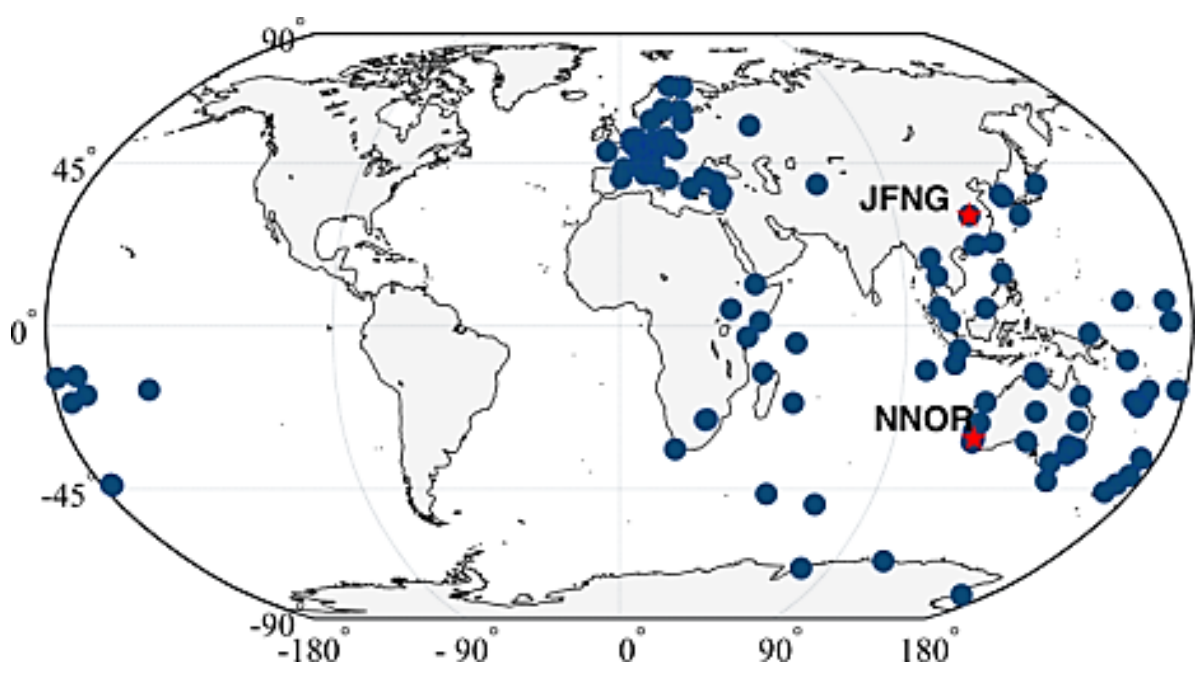

Fig. 2 List of MGEX stations with BeiDou observations on DOY 299, 2017.

the two papers for more details. We note that a conservative threshold is set in pre-processing step considering the processing of 5 minutes interval observations and the multi-frequency observations. But to ensure the quality in the PPP or other related applications, more precise outlier detection is implemented based on post-fit residual screening.

Apart from the quality of individual pseudorange and phase observations, G-Nut/Anubis estimates coordinates of the receiver using the pseudo-range ionosphere-free combinations using the standard point positioning method, but note that the timing group delay (TGD) is not corrected in the G-Nut package. The receiver position is currently estimated as kinematic parameter with the variance of $10000 \mathrm{~m}$ for each GNSS separately using a common sampling rate of 15 minutes. Besides, the troposphere is modeled as a random-walk process and estimated together with the coordinates, with its initial random-walk variances set to $10 \mathrm{~cm}$ and random-walk process noise is set to $3 \mathrm{~mm}$ per sqrt(hour).

\section{EXPERIMENTS DESIGN AND INITIAL RESULTS}

To evaluate the quality of BeiDou/GPS observations, the experimental period starting from day 001, 2013 to day 300, 2017 are selected. The observations are processed using the G-Nut/Anubis package. Since we focused on analyzing the long-term variations of the observation quality indicators, the data are processed with a 7-day interval. All the daily observations are processed with a sample of 5 minutes. In the case of raw observation evaluation, the indicators on each band are calculated separately. In the case of SPP experiments, the broadcast ephemerids are used and both coordinates and tropospheric delays in zenith are estimated as unknown parameters. The statistics results are calculated over all valid epochs in the day.

\section{DATE AVAILABILITY EVALUATION}

Figure 2 presents the MGEX stations that tracking BeiDou signal on DOY (Day Of Year) 299, 2017. Only the stations which can provide a minimum of 6-hour observations are selected. Such stations are usable for BeiDou precise orbit determination and clock estimation. It is observed that the stations are distributed unevenly, which will affect the precision of products at a global scale. Statistical on the receiver types show that four receiver types are commonly used in Multi-GNSS Experiment (MGEX) stations, i.e. LEICA GR*, SEPT POLARX*, JAVAD TRE_* and TRIMBLE NETR9, where '*' represents different versions. For the data evaluation, the MGEX station JFNG, which adopted the TRIMBLE NETR9 receiver, is used. Besides, station NNOR, which equipped with receiver SEPT POLARX4, is used for comparative analysis.

\section{DATE COMPLETENESS EVALUATION}

Elevation angle cut-off 0 degree is commonly recommended for data tracking at all scientific stations. Therefore, completeness indicator of the observation data above 0 degrees is selected. Figure 3 shows the completeness ratios on each band for station JFNG and NNOR. Since the third frequency is not available for NNOR, only the dual-frequency results are plotted. Minor difference is observed in completeness of phase and code observations, thus the phase ratios are plotted only. It is observed from the left figure (station JFNG) that the ratios on the first two frequencies are higher than that of the third frequency for GPS observations before 2014. After that, the GPS observation completeness in different frequency gradually reaches the same amount. In comparison, the same completeness ratios are observed for BeiDou at the first two frequencies. However, loss of the tracking BeiDou third frequency observables is observed during the period of 
$2015 / 06 / 23$ to $2015 / 08 / 18$. This is caused by the change of receiver settings during the update of new receiver firmware, which has been confirmed by the host agency.

Moreover, it is observed that ratios for BeiDou are always higher than that of the GPS. This is mainly due to the use of GEO and IGSO satellite observations, which make the averaged BeiDou satellite elevations higher than that of GPS. A significant degradation of completeness ratio for both GPS and BeiDou is observed between 2014/08/05 and 2014/08/12; this is mainly due to the inconsistency between observation header information and the actual observation records. More importantly, a gradually decline trend is observed for the GPS data completeness. The similar trend can also be observed for station NNOR. To further identify the trend is caused by the receiver- or constellation-specific reasons. We additionally analyze the observations from station ALIC, equipped with LEICA GR25 receiver, and stations FFMJ, equipped with JAVAD receivers after 02 April, 2014. The completeness ratios on band L1 and L2 are also shown in Figure 3. As we can observe, the degradation trend is not observed on station FFMJ, therefore, the cause for the change might be attributed to the receiver specific reason. As the completeness indicator illustrates the comprehensive performance of the receiver and the satellite signals, users can utilize the indicator to identify the receiver-/constellation-specific changes.

\section{PSEUDORANGE MULTIPATH EVALUATION}

Pseudorange multipath and signal noises represent the largest remaining unmanaged error source in PPP. Figure 4 shows the root mean square (RMS) of daily multipath series for each frequency at two selected stations. The vertical grey line indicates the change of receive firmware update, which is coming from the IGS station log files. For the GPS, we can observe that the multipath RMS on L1 frequency of station JFNG (left figure) is the smallest, followed by the multipath on the L2 and L3 frequency. But on station NNOR, the multipath on the $\mathrm{L} 1$ is larger than that of the L2 observations.

When comparing between GPS and BeiDou multipath RMS, the BeiDou have smaller RMS than GPS on station JFNG on all frequencies. For station NNOR, the multipath on the first BeiDou frequency is smaller than that of the GPS; but on the second frequency, the BeiDou suffers from much larger multipath. There are certainly signal-specific multipath characteristics because different signals are designed to be less or more resistance against the multipath. However, an actual multipath also depends on the environment and is strongly environment/signal-/receiver-specific. This is why we observe the actual multipath characteristics vary with frequencies and stations. We can also observe a significant multipath RMS jump on station JFNG for signal BDSM7I between 2013/09/03 and 2013/11/12. An observation data gap, as well a receiver firmware update occurred in this period. In comparison, the jump is not observed for station NNOR; this indicates that the jump on JFNG is caused by the firmware update. Another multipath jump occurs at 2017/07/11 for GPSM2X and BDSM7I signal. An update of receiver firmware is also checked. The results indicated the RMS can be correlated with the receiver firmware too. Considering the multipath series has been widely used to decrease the code noise by means of the sidereal filtering, therefore, the related applications should pay special attention to the change of the receiver types and receiver firmware.

\section{CARRIER PHASE CYCLE SLIP EVALUATION}

The cycle slip counts on each frequency of GPS and BeiDou observations are shown in Figure 5. It is observed that, for GPS observations, the observation type L1C is more affected by the cycle slips, whereas the L5X is less affected, which indicate the improvement of signal quality. In contrast, BeiDou observations are more affected by the cycle slips, which are mainly due to the poor quality of BeiDou C05 observations. Though no significant difference in cycle-slip counts is observed for BeiDou signal on different frequencies, there is a short period with the counts of BeiDou observations is decreased significantly. Simultaneously, a loss of tracking of BDSL6I signal is observed during the period. Therefore, we judge that the cycle slips for BeiDou $\mathrm{C} 05$ is mainly caused by the third frequency observable. Besides, the slip counts for station NNOR are analysed to confirm the conclusion.

\section{COMPREHENSIVE SPP EVALUATION}

The coordinate solutions from GPS and BeiDou SPP are compared with respect to the reference coordinates calculated from daily static PPP using GPS observations from DOY 299, 2017. The coordinates difference in north, east and up direction are shown in Figure 6. Robust outlier detection methods are used in the post-fit residual screening procedure. It is observed that the GPS positioning can achieve a relatively high accuracy compared to that of the BeiDou. Statistical results show that the averaged position RMS for GPS is $0.28 \mathrm{~m}, 0.20 \mathrm{~m}$ and $0.46 \mathrm{~m}$ for north, east and up direction, whereas the accuracy is $1.78 \mathrm{~m}, 3.40 \mathrm{~m}$ and $1.39 \mathrm{~m}$ for BeiDou. The systematic biases and large RMS in the east direction is mainly due to the distribution of BeiDou satellites. Positioning accuracy degradation is observed for BeiDou after 2016/05/17. Analysis shows that this is caused by the BeiDou satellite $\mathrm{C} 13 / \mathrm{C} 15$. They are actually the same satellite as the PRN of the original C15 satellite was changed to C13 on 11 December 2016. After removing the both corresponding satellite PRNs (C13 and C15), systematic biases fully disappeared. Detailed analysis illustrates that the problem was mainly caused by large errors in satellite orbits. 
The precision of the SPP is dominated by the pseudorange noise, multipath and quality of broadcast ephemeris and satellite geometry. Kazmierski et al. (2018) illustrated that the noise standard deviations for BeiDou and GPS pseudorange observables are at the same level. From Figure 4 we can observe that no significant differences in multipath errors exist between GPS and BeiDou observations at station JFNG. The poor SPP precision for BeiDou can be thus attributed to the large broadcast ephemeris errors. To illustrate the precision of broadcast ephemeris, we develop a G-Nut/Aset tool for the evaluation of precise products and navigation data. The broadcast ephemerides downloaded from MGEX are compared to the final orbit provided by the GFZ. The satellite position and clock correction is calculated every 5 minutes, and the orbit differences in radial, along track, and cross track component are computed. The RMS is calculated every 1 hour. Only radial orbit and clock errors are analysed considering their large effects to the positioning. Due to the lack of final orbit from GFZ, there is no comparison prior 2014. For better visualization, the orbit errors exceeding $4 \mathrm{~m}$ for GPS and $8 \mathrm{~m}$ for BeiDou are plotted on the $y$-axis at $4 \mathrm{~m}$ and $8 \mathrm{~m}$ values, respectively.

Figure 7 shows the radial orbit errors for different BeiDou satellite types. Different colours represent different satellites. Gradually improvement of $\mathrm{GEO} / \mathrm{IGSO} / \mathrm{MEO}$ orbit precision is observed, which might be attributed to the increased number of usable tracking stations. The orbit errors within the same constellations are of the same magnitude, but the orbit errors for BeiDou MEO and IGSO satellites are slightly better than GEO satellites. However, the BeiDou clock errors plotted in Figure 8 show rather different characteristics. Largest clock errors are observed for C13 and C15 PRNs, which can explain its adverse effect to SPP results. Large clock error is also observed for satellite $\mathrm{C} 01$ and the MEO constellation satellites. This illustrates that the clock errors does not depend on the constellation type only. Note that since the TGD is not considered in G-Nut library currently, the clock evaluation also ignores its effects.

Finally, the precision of GPS ephemeris is also analysed, although not presented. A gradual improvement in orbit errors is observed, which is mainly attributed to the update of old satellite types to the new Block IIF satellites. Generally, outliers are detected for both GPS and BeiDou broadcast ephemerids even if the satellite status indicated as healthy; the BeiDou is more frequently affected by the outliers. The broadcast ephemeris should be carefully detected and monitored for real-time users, which is another potential application of the G-Nut library.

\section{CONCLUSION AND OUTLOOK}

The G-Nut/Anubis software package is introduced for the quality monitoring of BeiDou/GPS observations. Quantitative indicators show that the completeness of BeiDou phase and code observations is higher than that of the GPS observations. This is mainly due to the contribution of GEO and IGSO observations. In terms of quality, no significant correlation is observed between the multipath and the bandwidth, but higher between multipath and receiver type and firmware. Users applying the sidereal filter to reduce the multipath error should thus give attention also to the firmware updates. In terms of carrier phase quality, the BeiDou C05 satellite is more affected by many cycle slips, in particular on the third frequency.

Comprehensive SPP evaluation shows that the positioning accuracy for BeiDou is worse than that of GPS, which is caused by the large broadcast orbit errors and still worse geometry of BeiDou constellations. More importantly, the BeiDou SPP accuracy is further degraded due to the poor clock precision in satellite $\mathrm{C} 13 / \mathrm{C} 15$. Orbit and clock evaluation show that the orbit errors are more related to the constellations, whereas the clock error not. Both GPS and BeiDou broadcast data suffers by outliers in case of satellites marked as healthy, however, more often it occurred for BeiDou. It is thus necessary to monitoring the broadcast orbit for real-time applications.

In 2015, China started to build up the BeiDou -3 . In addition to $\mathrm{B} 1$ and $\mathrm{B} 3$ signals, the BDS-3 satellites are also capable of transmitting several new navigation signals, namely $\mathrm{B} 1 \mathrm{C}, \mathrm{B} 2 \mathrm{a}$ and $\mathrm{B} 3 \mathrm{~b}$. In the near future, more BeiDou -3 satellites, as well new GPS, GLONASS and Galileo satellites will be launched. It is promising that the software can be used to identify the benefits and defects of observations caused by new signals, receivers and environmentalspecific aspects. Apart from the selected indicator illustrated in our experiments, there are other indicators, such as number of data gaps, receiver jumps and signal-to-noise ratio and others, provided by the G-Nut/Anubis software. A detailed introduction can refer to the home page of GOP (http://software.pecny.cz/anubis/).

\section{ACKNOWLEDGEMENTS}

The G-Nut/Anubis software has been recently enhanced for the quality control indicators within the EU H2020 (EPOS-IP project, No. 676564). We also acknowledge the support of Ministry of Education, Youth and Sports of the Czech Republic (Project No. LO1506). We would like to thank two anonymous reviewers for their valuable suggestions and comments. The MGEX data were provided by the IGS.

\section{REFERENCES}

Douša, J., Václavovic, P., Zhao, L. and Kačmařík, M.: 2018, New adaptable all-in-one strategy for estimating advanced tropospheric parameters and using real-time orbits and clocks. Remote Sens., 10, 2, 232.

DOI: $10.3390 / \mathrm{rs} 10020232$ 
Dousa, J. and Elias, M.: 2014, An improved model for calculating tropospheric wet delay. Geophys. Res. Lett., 41, 4389-4397. DOI: 10.1002/2014GL060271.

Douša, J., Václavovic P. and Eliáš, M.: 2017, Tropospheric products of the second European GNSS reprocessing (1996-2014). Atmos. Meas. Tech., 10, 1-19. DOI: $10.5194 /$ amt-10-1-2017

Estey, L.H. and Meertens, C.M.: 1999, TEQC: The multipurpose toolkit for GPS/GLONASS data. GPS Solut., 3, 1, 42-49. DOI: 10.1007/p100012778

Geng, J. and Bock, Y.: 2013, Triple-frequency GPS precise point positioning with rapid ambiguity resolution. J. of Geod., 87, 5, 449-460. DOI: $10.1007 / \mathrm{s} 00190-013-0619-2$

Kačmařík, M., Douša, J, Dick, G., et al.: 2017, Intertechnique validation of tropospheric slant total delays. Atmos. Meas. Tech. Discuss., 1-38. DOI: 10.5194/amt-2016-372

Kazmierski, K., Hadas, T. and Sośnica, K.: 2018, Weighting of Multi-GNSS observations in real-time precise point positioning. Remote Sens., 10, 1, 84. DOI: $10.3390 /$ rs 10010084

Kim, M., Seo, J. and Lee, J.: 2014, A comprehensive method for GNSS data quality determination to improve ionospheric data analysis. Senzore. 14(12), 14971-14993. DOI: 10.3390/s140814971

Langley, R.B.: 1999, Dilution of precision. GPS world, 10, $5,52-59$.

Li, T., Wang, J. and Laurichesse, D.: 2013, Modeling and quality control for reliable precise point positioning integer ambiguity resolution with GNSS modernization. GPS Solut., 18, 3, 429-442. DOI: $10.1007 / \mathrm{s} 10291-013-0342-8$

Li, X., Zhang, X. and Guo, F.: 2014, Predicting atmospheric delays for rapid ambiguity resolution in precise point positioning. Adv. Space Res., 54, 5, 840-850. DOI: 10.1016/j.asr.2013.07.021

Nicolini, L. and Caporali, A.: 2018, Investigation on reference frames and time systems in multi-GNSS. Remote Sens., 10, 1, 80. DOI: 10.3390/rs10010080
Nischan, T.: 2016, GFZRNX - RINEX GNSS data conversion and manipulation toolbox (Version 1.05). GFZ Data Services.

DOI: 10.5880/GFZ.1.1.2016.002

Vaclavovic, P., Douša, J. and Gyori, G.: 2013, G-Nut software library - state of development and first results, Acta Geodyn. Geomater., 10, No. 4 (172), 431-436. DOI: 10.13168/AGG.2013.0042

Vaclavovic, P. and Douša, J.: 2015a, G-Nut/Anubis: Opensource tool for Multi-GNSS data monitoring with a multipath detection for new signals, Frequencies and Constellations. IAG 150 Years, 775-782. DOI: $10.1007 / 1345201597$

Vaclavovic, P. and Douša, J.: 2015b, Development towards advanced GNSS data quality monitoring, Družicové metody v geodézii a katastru, Sborník referátů, Brno, ECON publishing, s.r.o, 76-81.

Ye, S., Zhao, L., Song, J., Chen, D. and Jiang, W.: 2017, Analysis of estimated satellite clock biases and their effects on precise point positioning. GPS Solut., 22, 1. DOI: $10.1007 / \mathrm{s} 10291-017-0680-\mathrm{Z}$

Zhao, Q., Sun, B., Dai, Z., Hu, Z., Shi, C. and Liu, J.: 2014, Real-time detection and repair of cycle slips in triplefrequency GNSS measurements. GPS Solut., 19, 3, 381-391. DOI: 10.1007/s10291-014-0396-2

Zumberge, J.F., Heflin, M.B., Jefferson, D.C., Watkins, M.M., and Webb, F.H.: 1997, Precise point positioning for the efficient and robust analysis of GPS data from large networks. J. Geophys. Res., Solid Earth, 102, B3, 5005-5017.

DOI: $10.1029 / 96 j b 03860$ 



Fig. 3 Statistics on the completeness of phase observation on selected stations.


Fig. 4 RMS of code multipath on station JFNG (left) and NNOR (right). 

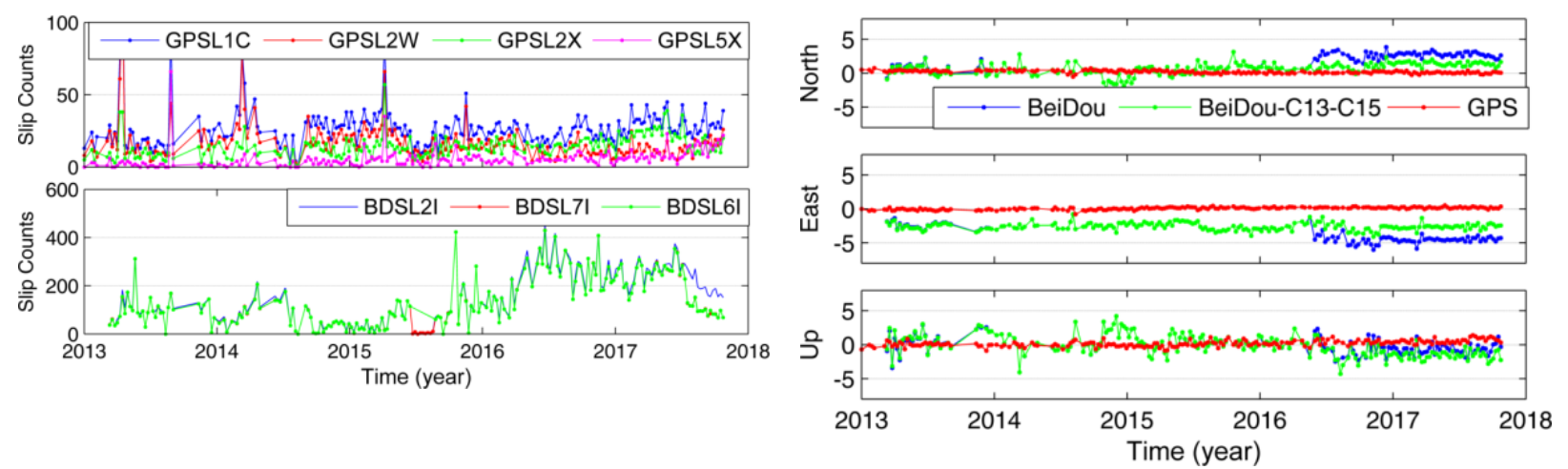

Fig. 5 Number of cycle slip counts on station JFNG.

Fig. 6 SPP positioning difference with respect to the references coordinates for GPS and BeiDou on station JFNG (unit: meters).



Individual orbit error for GEO

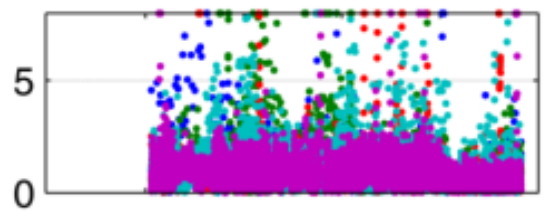

Individual orbit error for MEO



Fig. 7 Radial orbit errors from BeiDou broadcast ephemeris.

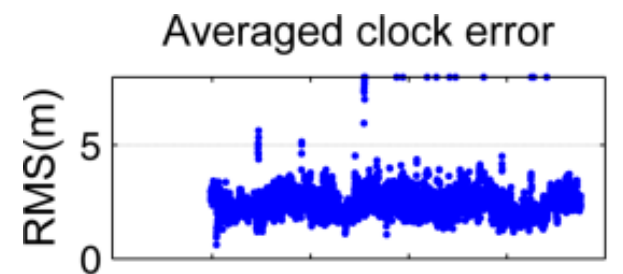

Individual clock error for IGSO



Individual clock error for GEO

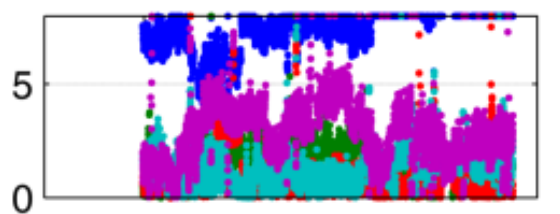

Individual clock error for MEO

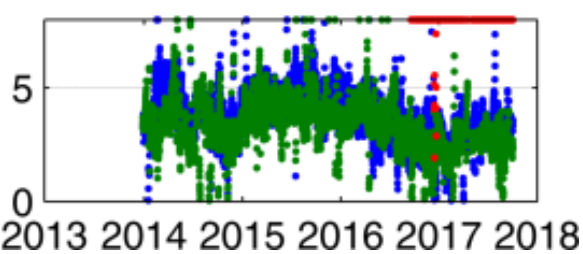

Fig. 8 Clock errors from BeiDou broadcast ephemeris. 